\title{
Systematic Review of Physiologic Monitor Alarm Characteristics and Pragmatic Interventions to Reduce Alarm Frequency
}

\author{
Christine Weirich Paine, MPH ${ }^{1,2}$, Veena V. Goel, MD,3,5,6, Elizabeth Ely, PhD, RN , Christopher D. Stave, MLS ${ }^{8}$, \\ Shannon Stemler, BA ${ }^{1}$, Miriam Zander, BA ${ }^{1}$, Christopher P. Bonafide, MD, MSCE $E^{1,9,10,11 \star}$
}

\begin{abstract}
'Division of General Pediatrics, The Children's Hospital of Philadelphia, Philadelphia, Pennsylvania; 2PolicyLab, The Children's Hospital of Philadelphia, Philadelphia, Pennsylvania; ' ${ }^{2}$ Department of Pediatrics, Stanford University School of Medicine, Stanford, California; ' ${ }^{4}$ Division of Systems Medicine, Stanford University School of Medicine, Stanford, California; ${ }^{5}$ Department of Clinical Informatics, Stanford Children's Health, Stanford, California; ${ }^{6}$ Division of Pediatric Hospital Medicine, Lucile Packard Children's Hospital Stanford, Palo Alto, California; ${ }^{7}$ Center for Pediatric Nursing Research and Evidence-Based Practice, The Children's Hospital of Philadelphia, Philadelphia, Pennsylvania; 'Lane Medical Library, Stanford University School of Medicine, Stanford, California; 'Department of Biomedical and Health Informatics, The Children's Hospital of Philadelphia, Philadelphia, Pennsylvania; ${ }^{10}$ Center for Pediatric Clinical Effectiveness, The Children's Hospital of Philadelphia, Philadelphia, Pennsylvania; ${ }^{11}$ Department of Pediatrics, Perelman School of Medicine at the University of Pennsylvania, Philadelphia, Pennsylvania.
\end{abstract}

BACKGROUND: Alarm fatigue from frequent nonactionable physiologic monitor alarms is frequently named as a threat to patient safety.

PURPOSE: To critically examine the available literature relevant to alarm fatigue.

DATA SOURCES: Articles published in English, Spanish, or French between January 1980 and April 2015 indexed in PubMed, Cumulative Index to Nursing and Allied Health Literature, Scopus, Cochrane Library, Google Scholar, and ClinicalTrials.gov.

STUDY SELECTION: Articles focused on hospital physiologic monitor alarms addressing any of the following: (1) the proportion of alarms that are actionable, (2) the relationship between alarm exposure and nurse response time, and (3) the effectiveness of interventions in reducing alarm frequency.

DATA EXTRACTION: We extracted data on setting, collection methods, proportion of alarms determined to be actionable, nurse response time, and associations between interventions and alarm rates.
DATA SYNTHESIS: Our search produced 24 observational studies focused on alarm characteristics and response time and 8 studies evaluating interventions. Actionable alarm proportion ranged from $<1 \%$ to $36 \%$ across a range of hospital settings. Two studies showed relationships between high alarm exposure and longer nurse response time. Most intervention studies included multiple components implemented simultaneously. Although studies varied widely, and many had high risk of bias, promising but still unproven interventions include widening alarm parameters, instituting alarm delays, and using disposable electrocardiographic wires or frequently changed electrocardiographic electrodes.

CONCLUSIONS: Physiologic monitor alarms are commonly nonactionable, and evidence supporting the concept of alarm fatigue is emerging. Several interventions have the potential to reduce alarms safely, but more rigorously designed studies with attention to possible unintended consequences are needed. Journal of Hospital Medicine 2016;11:136-144. (c) 2015 Society of Hospital Medicine
Clinical alarm safety has become a recent target for improvement in many hospitals. In 2013, The Joint Commission released a National Patient Safety Goal prompting accredited hospitals to establish alarm safety as a hospital priority, identify the most important alarm signals to manage, and, by 2016, develop policies and procedures that address alarm management. ${ }^{1}$ In addition, the Emergency Care Research Institute has named alarm hazards the top health technology hazard each year since $2012 .^{2}$

\footnotetext{
${ }^{\star}$ Address for correspondence and reprint requests: Christopher $\mathrm{P}$. Bonafide, MD, MSCE, The Children's Hospital of Philadelphia, 3401 Civic Center Blvd., Philadelphia, PA 19104; Telephone: 267-426-2901; E-mail: bonafide@email.chop.edu

Additional Supporting Information may be found in the online version of this article.

Received: July 18, 2015; Revised: October 1, 2015; Accepted: October 6, 2015

2015 Society of Hospital Medicine DOI 10.1002/jhm.2520

Published online in Wiley Online Library (Wileyonlinelibrary.com).
}

The primary arguments supporting the elevation of alarm management to a national hospital priority in the United States include the following: (1) clinicians rely on alarms to notify them of important physiologic changes, (2) alarms occur frequently and usually do not warrant clinical intervention, and (3) alarm overload renders clinicians unable to respond to all alarms, resulting in alarm fatigue: responding more slowly or ignoring alarms that may represent actual clinical deterioration. ${ }^{3,4}$ These arguments are built largely on anecdotal data, reported safety event databases, and small studies that have not previously been systematically analyzed.

Despite the national focus on alarms, we still know very little about fundamental questions key to improving alarm safety. In this systematic review, we aimed to answer 3 key questions about physiologic monitor alarms: (1) What proportion of alarms warrant attention or clinical intervention (ie, "actionable" alarms), and how does this proportion vary between adult and 
pediatric populations and between intensive care unit (ICU) and ward settings? (2) What is the relationship between alarm exposure and clinician response time? (3) What interventions are effective in reducing the frequency of alarms?

We limited our scope to monitor alarms because few studies have evaluated the characteristics of alarms from other medical devices, and because missing relevant monitor alarms could adversely impact patient safety.

\section{METHODS}

We performed a systematic review of the literature in accordance with the Meta-Analysis of Observational Studies in Epidemiology guidelines ${ }^{5}$ and developed this manuscript using the Preferred Reporting Items for Systematic Reviews and Meta-Analyses (PRISMA) statement. ${ }^{6}$

\section{Eligibility Criteria}

With help from an experienced biomedical librarian (C.D.S.), we searched PubMed, the Cumulative Index to Nursing and Allied Health Literature, Scopus, Cochrane Library, ClinicalTrials.gov, and Google Scholar from January 1980 through April 2015 (see Supporting Information in the online version of this article for the search terms and queries). We hand searched the reference lists of included articles and reviewed our personal libraries to identify additional relevant studies.

We included peer-reviewed, original research studies published in English, Spanish, or French that addressed the questions outlined above. Eligible patient populations were children and adults admitted to hospital inpatient units and emergency departments (EDs). We excluded alarms in procedural suites or operating rooms (typically responded to by anesthesiologists already with the patient) because of the differences in environment of care, staff-to-patient ratio, and equipment. We included observational studies reporting the actionability of physiologic monitor alarms (ie, alarms warranting special attention or clinical intervention), as well as nurse responses to these alarms. We excluded studies focused on the effects of alarms unrelated to patient safety, such as families' and patients' stress, noise, or sleep disturbance. We included only intervention studies evaluating pragmatic interventions ready for clinical implementation (ie, not experimental devices or software algorithms).

\section{Selection Process and Data Extraction}

First, 2 authors screened the titles and abstracts of articles for eligibility. To maximize sensitivity, if at least 1 author considered the article relevant, the article proceeded to full-text review. Second, the full texts of articles screened were independently reviewed by 2 authors in an unblinded fashion to determine their eligibility. Any disagreements concerning eligibility were resolved by team consensus. To assure consistency in eligibility determinations across the team, a core group of the authors (C.W.P, C.P.B., E.E., and V.V.G.) held a series of meetings to review and discuss each potentially eligible article and reach consensus on the final list of included articles. Two authors independently extracted the following characteristics from included studies: alarm review methods, analytic design, fidelity measurement, consideration of unintended adverse safety consequences, and key results. Reviewers were not blinded to journal, authors, or affiliations.

\section{Synthesis of Results and Risk Assessment}

Given the high degree of heterogeneity in methodology, we were unable to generate summary proportions of the observational studies or perform a metaanalysis of the intervention studies. Thus, we organized the studies into clinically relevant categories and presented key aspects in tables. Due to the heterogeneity of the studies and the controversy surrounding quality scores, ${ }^{5}$ we did not generate summary scores of study quality. Instead, we evaluated and reported key design elements that had the potential to bias the results. To recognize the more comprehensive studies in the field, we developed by consensus a set of characteristics that distinguished studies with lower risk of bias. These characteristics are shown and defined in Table 1.

For the purposes of this review, we defined nonactionable alarms as including both invalid ("false") alarms that do not that accurately represent the physiologic status of the patient and alarms that are valid but do not warrant special attention or clinical intervention ("nuisance" alarms). We did not separate out invalid alarms due to the tremendous variation between studies in how validity was measured.

\section{RESULTS \\ Study Selection}

Search results produced 4629 articles (see the flow diagram in the Supporting Information in the online version of this article), of which 32 articles were eligible: 24 observational studies describing alarm characteristics and 8 studies describing interventions to reduce alarm frequency.

\section{Observational Study Characteristics}

Characteristics of included studies are shown in Table 1. Of the 24 observational studies, ${ }^{7-30} 15$ included adult patients, ${ }^{7-21} 7$ included pediatric patients, ${ }^{22-28}$ and 2 included both adult and pediatric patients. ${ }^{29,30}$ All were single-hospital studies, except for 1 study by Chambrin and colleagues ${ }^{10}$ that included 5 sites. The number of patient-hours examined in each study ranged from 60 to $113,880 .^{7-11,13-16,18-27,29,30}$ Hospital settings included ICUs $(\mathrm{n}=16),{ }^{9-11,13,14,16-19,22-27,29}$ general wards $(\mathrm{n}=$ 5), ${ }^{12,15,20,22,28}$ EDs $(\mathrm{n}=2),{ }^{7,21}$ postanesthesia care unit 


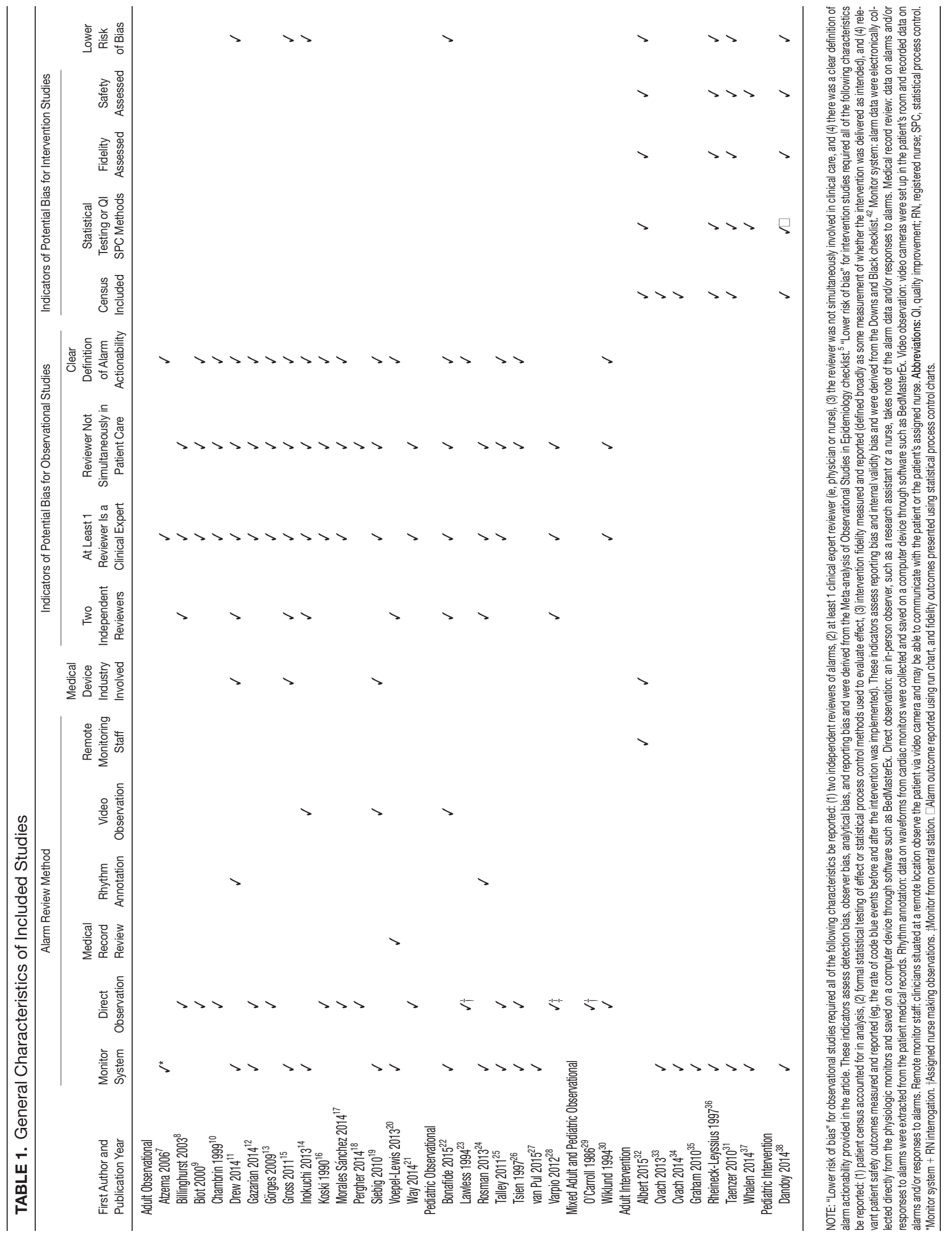


(PACU) $(\mathrm{n}=1),{ }^{30}$ and cardiac care unit (CCU) $(\mathrm{n}=$ 1). ${ }^{8}$ Studies varied in the type of physiologic signals recorded and data collection methods, ranging from direct observation by a nurse who was simultaneously caring for patients ${ }^{29}$ to video recording with expert review. ${ }^{14,19,22}$ Four observational studies met the criteria for lower risk of bias. ${ }^{11,14,15,22}$

\section{Intervention Study Characteristics}

Of the 8 intervention studies, 7 included adult patients, ${ }^{31-37}$ and 1 included pediatric patients. ${ }^{38}$ All were single-hospital studies; 6 were quasiexperimental ${ }^{31,33-35,37,38}$ and 2 were experimental. ${ }^{32,36}$ Settings included progressive care units $(\mathrm{n}=3),{ }^{33-35}$ CCUs $(\mathrm{n}=3),{ }^{32,33,37}$ wards $(\mathrm{n}=2),{ }^{31,38}$ PACU $(\mathrm{n}=1),{ }^{36}$ and a step-down unit $(\mathrm{n}=1){ }^{32}$ All except 1 study $^{32}$ used the monitoring system to record alarm data. Several studies evaluated multicomponent interventions that included combinations of the following: widening alarm parameters, ${ }^{31,35-38}$ instituting alarm delays, ${ }^{31,34,36,38}$ reconfiguring alarm acuity, ${ }^{35,37}$ use of secondary notifications, ${ }^{34}$ daily change of electrocardiographic electrodes or use of disposable electrocardiographic wires, ${ }^{32,33,38}$ universal monitoring in high-risk populations, ${ }^{31}$ and timely discontinuation of monitoring in low-risk populations. ${ }^{38}$ Four intervention studies met our prespecified lower risk of bias criteria. $^{31,32,36,38}$

\section{Proportion of Alarms Considered Actionable}

Results of the observational studies are provided in Table 2. The proportion of alarms that were actionable was $<1 \%$ to $26 \%$ in adult ICU settings, ${ }^{9-11,13,14,16,17,19} 20 \%$ to $36 \%$ in adult ward settings, ${ }^{12,15,20} 17 \%$ in a mixed adult and pediatric PACU setting, ${ }^{30} 3 \%$ to $13 \%$ in pediatric ICU settings, ${ }^{22-26}$ and $1 \%$ in a pediatric ward setting. ${ }^{22}$

\section{Relationship Between Alarm Exposure and Response Time}

Whereas 9 studies addressed response time, ${ }^{8,12,17,18,20-22,27,28}$ only 2 evaluated the relationship between alarm burden and nurse response time. ${ }^{20,22}$ Voepel-Lewis and colleagues found that nurse responses were slower to patients with the highest quartile of alarms (57.6 seconds) compared to those with the lowest (45.4 seconds) or medium (42.3 seconds) quartiles of alarms on an adult ward $(P=$ 0.046). They did not find an association between false alarm exposure and response time. ${ }^{20}$ Bonafide and colleagues found incremental increases in response time as the number of nonactionable alarms in the preceding 120 minutes increased $(P<0.001$ in the pediatric ICU, $P=0.009$ on the pediatric ward). ${ }^{22}$

\section{Interventions Effective in Reducing Alarms}

Results of the 8 intervention studies are provided in Table 3. Three studies evaluated single interventions; $;^{32,33,36}$ the remainder of the studies tested inter- ventions with multiple components such that it was impossible to separate the effect of each component. Below, we have summarized study results, arranged by component. Because only 1 study focused on pediatric patients, ${ }^{38}$ results from pediatric and adult settings are combined.

Widening alarm parameter default settings was evaluated in 5 studies: ${ }^{31,35-38} 1$ single intervention randomized controlled trial (RCT), ${ }^{36}$ and 4 multipleintervention, quasi-experimental studies. ${ }^{31,35,37,38}$ In the RCT, using a lower $\mathrm{SpO}_{2}$ limit of $85 \%$ instead of the standard $90 \%$ resulted in $61 \%$ fewer alarms. In the 4 multiple intervention studies, 1 study reported significant reductions in alarm rates $(P<0.001),{ }^{37} 1$ study did not report preintervention alarm rates but reported a postintervention alarm rate of 4 alarms per patient-day, ${ }^{31}$ and 2 studies reported reductions in alarm rates but did not report any statistical testing. ${ }^{35,38}$ Of the 3 studies examining patient safety, 1 study with universal monitoring reported fewer rescue events and transfers to the ICU postimplementation, ${ }^{31}$ 1 study reported no missed acute decompensations, ${ }^{38}$ and 1 study (the RCT) reported significantly more true hypoxemia events $(P=0.001) .{ }^{36}$

Alarm delays were evaluated in 4 studies: ${ }^{31,34,36,38}$ 3 multiple-intervention, quasi-experimental studies $^{31,34,38}$ and 1 retrospective analysis of data from an RCT. ${ }^{36}$ One study combined alarm delays with widening defaults in a universal monitoring strategy and reported a postintervention alarm rate of 4 alarms per patient. ${ }^{31}$ Another study evaluated delays as part of a secondary notification pager system and found a negatively sloping regression line that suggested a decreasing alarm rate, but did not report statistical testing. ${ }^{34}$ The third study reported a reduction in alarm rates but did not report statistical testing. ${ }^{38}$ The RCT compared the impact of a hypothetical 15-second alarm delay to that of a lower $\mathrm{SpO}_{2}$ limit reduction and reported a similar reduction in alarms. ${ }^{36}$ Of the 4 studies examining patient safety, 1 study with universal monitoring reported improvements, ${ }^{31} 2$ studies reported no adverse outcomes, ${ }^{35,38}$ and the retrospective analysis of data from the RCT reported the theoretical adverse outcome of delayed detection of sudden, severe desaturations. ${ }^{36}$

Reconfiguring alarm acuity was evaluated in 2 studies, both of which were multiple-intervention quasiexperimental studies. ${ }^{35,37}$ Both showed reductions in alarm rates: 1 was significant without increasing adverse events $(P<0.001),{ }^{37}$ and the other did not report statistical testing or safety outcomes. ${ }^{35}$

Secondary notification of nurses using pagers was the main intervention component of 1 study incorporating delays between the alarms and the alarm pages. $^{34}$ As mentioned above, a negatively sloping regression line was displayed, but no statistical testing or safety outcomes were reported. 


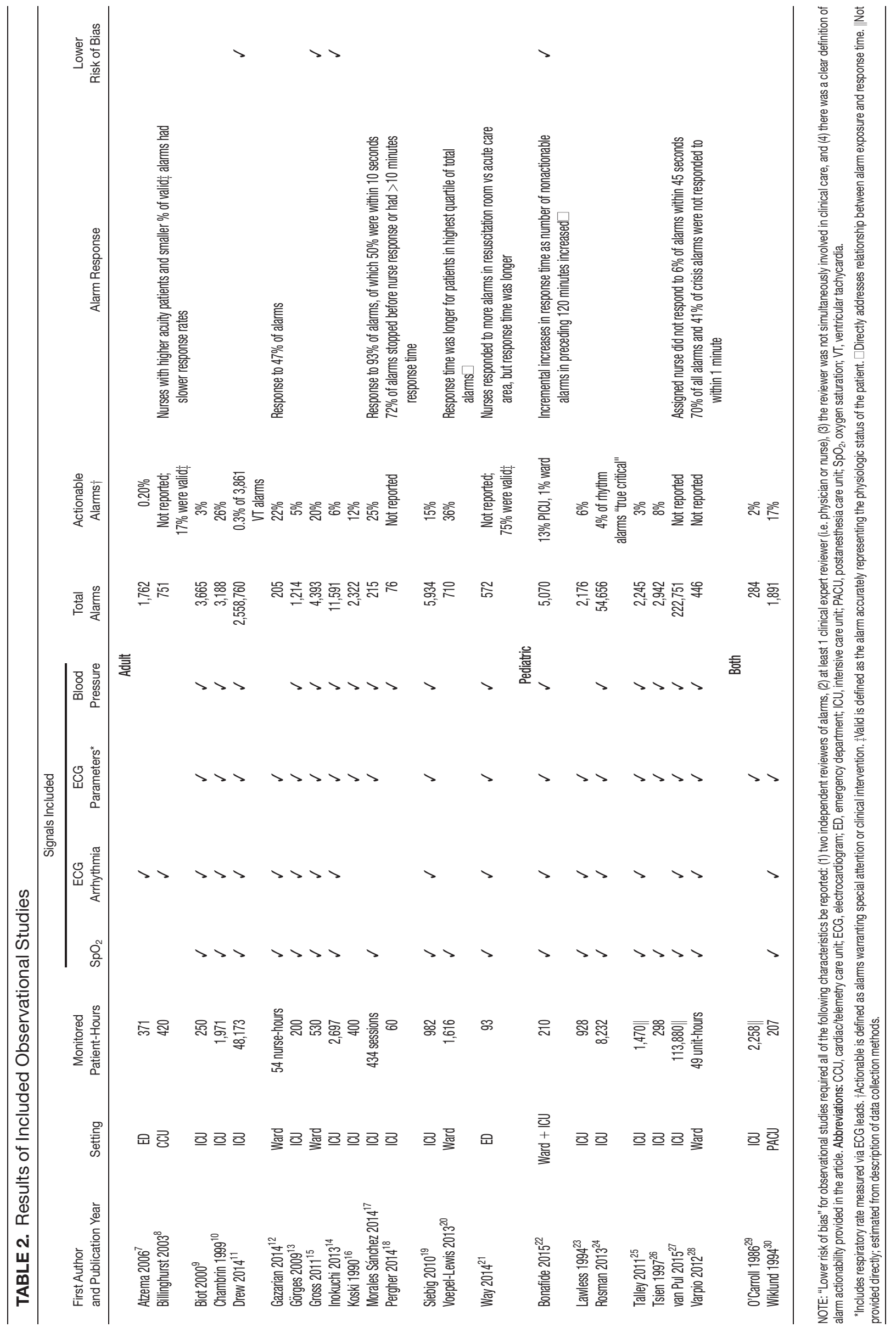


Review of Physiologic Monitor Alarms | Paine et al

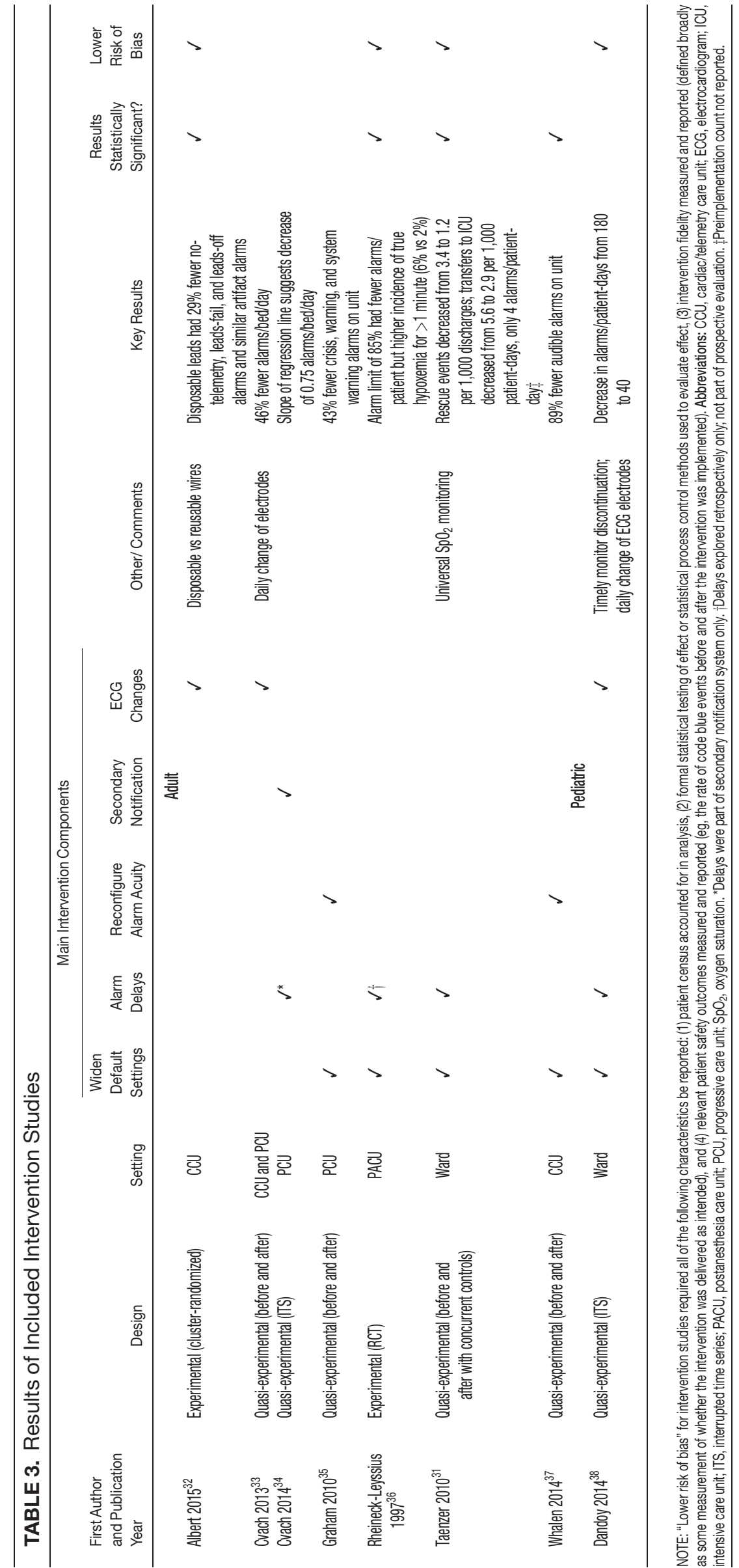


Disposable electrocardiographic lead wires or daily electrode changes were evaluated in 3 studies: $:^{32,33,38} 1$ single intervention cluster-randomized trial ${ }^{32}$ and 2 quasi-experimental studies. ${ }^{33,38}$ In the clusterrandomized trial, disposable lead wires were compared to reusable lead wires, with disposable lead wires having significantly fewer technical alarms for lead signal failures $(P=0.03)$ but a similar number of monitoring artifact alarms $(P=0.44) .{ }^{32}$ In a single-intervention, quasi-experimental study, daily electrode change showed a reduction in alarms, but no statistical testing was reported. ${ }^{33}$ One multipleintervention, quasi-experimental study incorporating daily electrode change showed fewer alarms without statistical testing. ${ }^{38}$ Of the 2 studies examining patient safety, both reported no adverse outcomes. ${ }^{32,38}$

\section{DISCUSSION}

This systematic review of physiologic monitor alarms in the hospital yielded the following main findings: (1) between $74 \%$ and $99 \%$ of physiologic monitor alarms were not actionable, (2) a significant relationship between alarm exposure and nurse response time was demonstrated in 2 small observational studies, and (3) although interventions were most often studied in combination, results from the studies with lower risk of bias suggest that widening alarm parameters, implementing alarm delays, and using disposable electrocardiographic lead wires and/or changing electrodes daily are the most promising interventions for reducing alarms. Only 5 of 8 intervention studies measured intervention safety and found that widening alarm parameters and implementing alarm delays had mixed safety outcomes, whereas disposable electrocardiographic lead wires and daily electrode changes had no adverse safety outcomes. ${ }^{29,30,34-36}$ Safety measures are crucial to ensuring the highest level of patient safety is met; interventions are rendered useless without ensuring actionable alarms are not disabled. The variation in results across studies likely reflects the wide range of care settings as well as differences in design and quality.

This field is still in its infancy, with 18 of the 32 articles published in the past 5 years. We anticipate improvements in quality and rigor as the field matures, as well as clinically tested interventions that incorporate smart alarms. Smart alarms integrate data from multiple physiologic signals and the patient's history to better detect physiologic changes in the patient and improve the positive predictive value of alarms. Academic-industry partnerships will be required to implement and rigorously test smart alarms and other emerging technologies in the hospital.

To our knowledge, this is the first systematic review focused on monitor alarms with specific review questions relevant to alarm fatigue. Cvach recently published an integrative review of alarm fatigue using research published through $2011 .^{39}$ Our review builds upon her work by contributing a more extensive and systematic search strategy with databases spanning nursing, medicine, and engineering, including additional languages, and including newer studies published through April 2015. In addition, we included multiple cross-team checks in our eligibility review to ensure high sensitivity and specificity of the resulting set of studies.

Although we focused on interventions aiming to reduce alarms, there has also been important recent work focused on reducing telemetry utilization in adult hospital populations as well as work focused on reducing pulse oximetry utilization in children admitted with respiratory conditions. Dressler and colleagues reported an immediate and sustained reduction in telemetry utilization in hospitalized adults upon redesign of cardiac telemetry order sets to include the clinical indication, which defaulted to the American Heart Association guideline-recommended telemetry duration. ${ }^{40}$ Instructions for bedside nurses were also included in the order set to facilitate appropriate telemetry discontinuation. Schondelmeyer and colleagues reported reductions in continuous pulse oximetry utilization in hospitalized children with asthma and bronchiolitis upon introduction of a multifaceted quality improvement program that included provider education, a nurse handoff checklist, and discontinuation criteria incorporated into order sets. ${ }^{41}$

\section{Limitations of This Review and the Underlying Body of Work}

There are limitations to this systematic review and its underlying body of work. With respect to our approach to this systematic review, we focused only on monitor alarms. Numerous other medical devices generate alarms in the patient-care environment that also can contribute to alarm fatigue and deserve equally rigorous evaluation. With respect to the underlying body of work, the quality of individual studies was generally low. For example, determinations of alarm actionability were often made by a single rater without evaluation of the reliability or validity of these determinations, and statistical testing was often missing. There were also limitations specific to intervention studies, including evaluation of nongeneralizable patient populations, failure to measure the fidelity of the interventions, inadequate measures of intervention safety, and failure to statistically evaluate alarm reductions. Finally, though not necessarily a limitation, several studies were conducted by authors involved in or funded by the medical device industry. ${ }^{11,15,19,31,32}$ This has the potential to introduce bias, although we have no indication that the quality of the science was adversely impacted.

Moving forward, the research agenda for physiologic monitor alarms should include the following: (1) more intensive focus on evaluating the relationship between alarm exposure and response time with 
analysis of important mediating factors that may promote or prevent alarm fatigue, (2) emphasis on studying interventions aimed at improving alarm management using rigorous designs such as clusterrandomized trials and trials randomized by individual participant, (3) monitoring and reporting clinically meaningful balancing measures that represent unintended consequences of disabling or delaying potentially important alarms and possibly reducing the clinicians' ability to detect true patient deterioration and intervene in a timely manner, and (4) support for transparent academic-industry partnerships to evaluate new alarm technology in real-world settings. As evidence-based interventions emerge, there will be new opportunities to study different implementation strategies of these interventions to optimize effectiveness.

\section{CONCLUSIONS}

The body of literature relevant to physiologic monitor alarm characteristics and alarm fatigue is limited but growing rapidly. Although we know that most alarms are not actionable and that there appears to be a relationship between alarm exposure and response time that could be caused by alarm fatigue, we cannot yet say with certainty that we know which interventions are most effective in safely reducing unnecessary alarms. Interventions that appear most promising and should be prioritized for intensive evaluation include widening alarm parameters, implementing alarm delays, and using disposable electrocardiographic lead wires and changing electrodes daily. Careful evaluation of these interventions must include systematically examining adverse patient safety consequences.

Acknowledgements: The authors thank Amogh Karnik and Micheal Sellars for their technical assistance during the review and extraction process.

Disclosures: Ms. Zander is supported by the Society of Hospital Medicine Student Hospitalist Scholar Grant. Dr. Bonafide and Ms. Stemler are supported by the National Heart, Lung, and Blood Institute of the National Institutes of Health under award number K23HL116427. The content is solely the responsibility of the authors and does not necessarily represent the official views of the National Institutes of Health. The authors report no conflicts of interest.

\section{References}

1. National Patient Safety Goals Effective January 1, 2015. The Joint Commission Web site. http://www.jointcommission.org/assets/1/6/ 2015_NPSG_HAP.pdf. Accessed July 17, 2015.

2. ECRI Institute. 2015 Top 10 Health Technology Hazards. Available at: https://www.ecri.org/Pages/2015-Hazards.aspx. Accessed June 23, 2015.

3. Sendelbach S, Funk M. Alarm fatigue: a patient safety concern. AACN Adv Crit Care. 2013;24(4):378-386.

4. Chopra V, McMahon LF Jr. Redesigning hospital alarms for patient safety: alarmed and potentially dangerous. JAMA. 2014;311(12): 1199-1200.

5. Stroup DF, Berlin JA, Morton SC, et al. Meta-analysis of observational studies in epidemiology: a proposal for reporting. Meta-analysis Of Observational Studies in Epidemiology (MOOSE) Group. JAMA. 2000;283(15):2008-2012.

6. Moher D, Liberati A, Tetzlaff J, Altman DG; PRISMA Group. Preferred reporting items for systematic reviews and meta- analyses: the PRISMA statement. Ann Intern Med. 2009;151(4):264269, W64.

7. Atzema C, Schull MJ, Borgundvaag B, Slaughter GRD, Lee CK. ALARMED: adverse events in low-risk patients with chest pain receiving continuous electrocardiographic monitoring in the emergency department. A pilot study. Am J Emerg Med. 2006;24:62-67.

8. Billinghurst F, Morgan B, Arthur HM. Patient and nurse-related implications of remote cardiac telemetry. Clin Nurs Res. 2003;12(4): 356-370.

9. Biot L, Carry PY, Perdrix JP, Eberhard A, Baconnier P. Clinical evaluation of alarm efficiency in intensive care [in French]. Ann Fr Anesth Reanim. 2000;19:459-466.

10. Chambrin MC, Ravaux P, Calvelo-Aros D, Jaborska A, Chopin C, Boniface B. Multicentric study of monitoring alarms in the adult intensive care unit (ICU): a descriptive analysis. Intensive Care Med. $1999 ; 25: 1360-1366$.

11. Drew BJ, Harris P, Zègre-Hemsey JK, et al. Insights into the problem of alarm fatigue with physiologic monitor devices: a comprehensive observational study of consecutive intensive care unit patients. PloS One. 2014;9(10):e110274.

12. Gazarian PK. Nurses' response to frequency and types of electrocardiography alarms in a non- critical care setting: a descriptive study. Int J Nurs Stud. 2014;51(2):190-197.

13. Görges M, Markewitz BA, Westenskow DR. Improving alarm performance in the medical intensive care unit using delays and clinical context. Anesth Analg. 2009;108:1546-1552.

14. Inokuchi R, Sato H, Nanjo Y, et al. The proportion of clinically relevant alarms decreases as patient clinical severity decreases in intensive care units: a pilot study. BMJ Open. 2013;3(9):e003354e003354.

15. Gross B, Dahl D, Nielsen L. Physiologic monitoring alarm load on medical/surgical floors of a community hospital. Biomed Instrum Technol. 2011;45:29-36.

16. Koski EM, Mäkivirta A, Sukuvaara T, Kari A. Frequency and reliability of alarms in the monitoring of cardiac postoperative patients. Int $J$ Clin Monit Comput. 1990;7(2):129-133.

17. Morales Sánchez C, Murillo Pérez MA, Torrente Vela S, et al. Audit of the bedside monitor alarms in a critical care unit [in Spanish]. Enferm Intensiva. 2014;25(3):83-90.

18. Pergher AK, da Silva RCL. Stimulus-response time to invasive blood pressure alarms: implications for the safety of critical-care patients. Rev Gaúcha Enferm. 2014;35(2):135-141.

19. Siebig S, Kuhls S, Imhoff M, Gather U, Scholmerich J, Wrede CE. Intensive care unit alarms- how many do we need? Crit Care Med. 2010;38:451-456.

20. Voepel-Lewis T, Parker ML, Burke CN, et al. Pulse oximetry desaturation alarms on a general postoperative adult unit: a prospective observational study of nurse response time. Int J Nurs Stud. 2013; 50(10):1351-1358.

21. Way RB, Beer SA, Wilson SJ. Whats that noise? Bedside monitoring in the Emergency Department. Int Emerg Nurs. 2014;22(4):197201.

22. Bonafide CP, Lin R, Zander M, et al. Association between exposure to nonactionable physiologic monitor alarms and response time in a children's hospital. J Hosp Med. 2015;10(6):345-351.

23. Lawless ST. Crying wolf: false alarms in a pediatric intensive care unit. Crit Care Med. 1994;22(6):981-985.

24. Rosman EC, Blaufox AD, Menco A, Trope R, Seiden HS. What are we missing? Arrhythmia detection in the pediatric intensive care unit. J Pediatr. 2013;163(2):511-514.

25. Talley LB, Hooper J, Jacobs B, et al. Cardiopulmonary monitors and clinically significant events in critically ill children. Biomed Instrum Technol. 2011;45(s1):38-45.

26. Tsien CL, Fackler JC. Poor prognosis for existing monitors in the intensive care unit. Crit Care Med. 1997;25:614-619.

27. van Pul C, VD Mortel H, VD Bogaart J, Mohns T, Andriessen P. Safe patient monitoring is challenging but still feasible in a neonatal intensive care unit with single family rooms. Acta Paediatr Oslo Nor 1992. 2015;104(6):e247-e254.

28. Varpio L, Kuziemsky C, Macdonald C, King WJ. The helpful or hindering effects of in-hospital patient monitor alarms on nurses: a qualitative analysis. CIN Comput Inform Nurs. 2012;30(4):210217.

29. O'Carroll T. Survey of alarms in an intensive therapy unit. Anaesthesia. 1986;41(7):742-744.

30. Wiklund L, Hök B, Ståhl K, Jordeby-Jönsson A. Postanesthesia monitoring revisited: frequency of true and false alarms from different monitoring devices. J Clin Anesth. 1994;6(3):182-188.

31. Taenzer AH, Pyke JB, McGrath SP, Blike GT. Impact of pulse oximetry surveillance on rescue events and intensive care unit transfers: a before-and-after concurrence study. Anesthesiology. 2010;112(2): 282-287.

32. Albert NM, Murray T, Bena JF, et al. Differences in alarm events between disposable and reusable electrocardiography lead wires. Am J Crit Care. 2015;24(1):67-74. 
33. Cvach MM, Biggs M, Rothwell KJ, Charles-Hudson C. Daily electrode change and effect on cardiac monitor alarms: an evidence-based practice approach. J Nurs Care Oual. 2013;28:265-271.

34. Cvach MM, Frank RJ, Doyle P, Stevens ZK. Use of pagers with an alarm escalation system to reduce cardiac monitor alarm signals. J Nurs Care Qual. 2014;29(1):9-18.

35. Graham KC, Cvach M. Monitor alarm fatigue: standardizing use of physiological monitors and decreasing nuisance alarms. Am J Crit Care. 2010;19:28-34.

36. Rheineck-Leyssius AT, Kalkman CJ. Influence of pulse oximeter lower alarm limit on the incidence of hypoxaemia in the recovery room. $\mathrm{BrJ}$ Anaesth. 1997;79(4):460-464.

37. Whalen DA, Covelle PM, Piepenbrink JC, Villanova KL, Cuneo CL, Awtry EH. Novel approach to cardiac alarm management on telemetry units. J Cardiovasc Nurs. 2014;29(5):E13-E22.
38. Dandoy CE, Davies SM, Flesch L, et al. A team-based approach to reducing cardiac monitor alarms. Pediatrics. 2014;134(6):e1686-e1694.

39. Cvach M. Monitor alarm fatigue: an integrative review. Biomed Instrum Technol. 2012;46(4):268-277.

40. Dressler R, Dryer MM, Coletti C, Mahoney D, Doorey AJ. Altering overuse of cardiac telemetry in non-intensive care unit settings by hardwiring the use of American Heart Association guidelines. JAMA Intern Med. 2014;174(11):1852-1854.

41. Schondelmeyer AC, Simmons JM, Statile AM, et al. Using quality improvement to reduce continuous pulse oximetry use in children with wheezing. Pediatrics. 2015;135(4):e1044-e1051.

42. Downs SH, Black N. The feasibility of creating a checklist for the assessment of the methodological quality both of randomised and non-randomised studies of health care interventions. I Epidemiol Community Health. 1998;52(6):377-384. 